\title{
The Foundation of the Fourth Law of Thermodynamics: Universe Dark Energy and its Nature: Can Dark Energy be Generated?
}

\author{
Murad Shibli \\ Mechatronics Engineering Department, Chairman \\ German Jordanian University, P.O. Box: 35247 Amman 11180 Jordan \\ Tel.: +962-6-5300-666 Ext. 212, Murad.Shibli@gju.edu.jo
}

\begin{abstract}
This paper presents a proposal of the fourth law of thermodynamics, the nature of the Universe Dark Energy and the potential to generate such an energy. Recent astronomical observation of Ia supernovae indicates that the Universe is expanding at accelerating rate and composed of around $70 \%$ dark energy; $25 \%$ dark matter; and $5 \%$ Hydrogen, Helium and stars. In this paper a physical interpretation of the dark energy is presented. This interpretation is based on geometric modeling of space-time as a phase fluid and the momentum generated by the time. In this modeling the time is considered to have a mechanical nature so that the momentum associated with it is equal to the negative of the universe total energy. Based on this analysis and by the virtue of the classical thermodynamics, the fourth law of thermodynamics is proposed to account for the dominating energy in the universe. It states that "Considering time as mechanical variable, for a closed system with moving boundaries composed of homogenous isotropic cosmic fluid, the system will have a negative pressure equal to the energy density that causes the system to expand at an accelerated rate. Moreover, the momentum associated with the time is equal to the negative of the system total energy". Such a law is very important to account for the dominating component of the universe, the $70 \%$ dark energy that is behind the accelerated expansion of the Universe. The possibility to generate such dark energy is then discussed utilizing macro or micro/nano systems. Finally, simulation results are demonstrated to verify the proposed results.
\end{abstract}

Keywords: Thermodynamics, Dark Energy, Fourth Law of Thermodynamics, Expansion of the Universe

\section{Introduction}

Thermodynamics can be defined as the science of energy. It is used to interpret all aspects of energy and energy transformations, including power generations, refrigeration, and relationships among the properties of matter. The basic principles of thermodynamics are summarized in four laws. Although the principles of thermodynamics have been existing since the creation of the universe, the first and second laws of thermodynamics emerged simultaneously in 1850s out of works of William Rankine, Rudolph Clasius and hLord Kelvin (formerly William Thomson).

The four laws of thermodynamics can be summarized in the following statements [1]:

- The Zeroth Law of Thermodynamics: If two bodies are in thermal equilibrium with a third body, they are also in thermal equilibrium with each other.
- The first Law of Thermodynamics: Energy is conserved and can only change its form.

- The Second Law of Thermodynamics (KelvinBlank Statement): It is impossible for any device that operates in a cycle to receive heat from a single reservoir and produce a net amount of work.

- The Third Law of Thermodynamics: The entropy of a pure crystalline substance at absolute zero temperature is zero.

Recent astronomical observations by the Supernova Cosmology Project, the High-z Supernova Search Team and cosmic microwave background (CMB) have provided strong evidence that our universe is not only expanding, but also expanding at an accelerating rate [310]. It was only in 1998 when dark energy proposed for the first time, after two groups of astronomers made a survey of exploding stars, or supernovas Ia, in a number of distant galaxies [3] [5]. These researchers found that the supernovas were dimmer than they should have been, and that meant they were farther away than they should have been. The only way for that to happen, the astronomers realized, was if the expansion of the universe had sped up at some time in the past, as well as accounting for a significant portion of a missing component in the universe. The only explanation is that there is a kind of force that has a strong negative pressure and acting outward in opposition to gravitational force at large scales which was proposed for the first time by Einstein in his General Relativity and given the name the cosmological constant Lambda [2]. This force is given the name Dark Energy, since it can not be observed or detected directly. These cosmological observations strongly suggest that the universe is dominated by a smoothly homogenous distributed dark energy component [11-21].

The dark energy that is causing the accelerated expansion of the universe - by any standard, it is more exotic and more poorly understood. What is known so far is that it contributes about $70 \%$ of the critical density and has a negative pressure [18-20]. According to the theory of General Relativity, the effect of such a negative pressure is qualitatively similar to a force acting in opposition to gravity at large scales. Invoking such an effect is currently the most popular method for explaining recent 
observations that the universe appears to be expanding at an accelerating rate, as well as accounting for a significant portion of the missing mass in the universe [19]. A Physical insight of dark energy and how to detect it using micro-nano space robotic system was proposed in [22-24].

Such an astronomical phenomena indicates that there is a need for the fourth law of thermodynamics to account for the most dominating part of the universe, the $70 \%$ Dark Energy. It is known to be very homogeneous, not very dense roughly around $10^{-29} \mathrm{gm} / \mathrm{cm}^{3}$ and interacts only with gravity. It is not very dense and roughly around $10^{-29} \mathrm{gm} / \mathrm{cm}^{3}$ [20]. It seems also that we live in a Universe which is full of energy every where and there is a potential to generate it and utilize it for the benifit of huminty. This potential is very important after the rising need for new sources of energy on earth. The property of dark enrgy to interact only with garvity indicates that dark enrgy can safely be used.

In this paper a physical interpretation of the dark energy is proposed. This physical insight is based on geometric modeling of space-time as a homogenous isotropic cosmic fluid and the momentum generated by the time. In this modeling the time is considered to have a mechanical nature such that the momentum associated with the time as a mechanical variable is equal to the negative of the system total energy. Based on this analysis the fourth law of thermodynamics is proposed. The statement of the fourth law will be proposed in the next section. Such a law is very important to describe the most dominating energy in nature $(70 \%$ dark energy) that is behind the accelerated expansion of the universe. Then it is followed by a discussion about the possibility to generate such an energy. Finally, simulation results are demonstrated to verify the proposed results.

\section{The Foundation of the Fourth Law of Thermodynamics, Dark Energy and its Nature}

Since thermodynamics is the science of energy, it is important to propose the fourth law to describe and account for the most dominating component of the universe, the dark energy. Astronomical observations of Supernovae 1a show that the universe is expanding at an accelerating rate. The only explanation is that there is a kind of force that has a strong negative pressure and acting outward in opposition to gravitational force at large scales. This force is given the name Dark Energy, since it can not be observed or detected directly.

Fourth Law of Thermodynamics Proposal: "Considering time as mechanical variable, for a closed system with moving boundaries composed of homogenous isotropic cosmic fluid, the system will have a negative pressure equal to the energy density that causes the system to expand at an accelerated rate. Moreover, the momentum associated with time is equal to the negative of the system total energy".

Proof 1 (Classical Thermodynamics): Since the universe is expanding, then its volume is increasing and its boundaries are moving. A closed system composed of homogenous isotropic cosmic fluid (space-time universe), with moving boundaries is then considered in this proof. In classical thermodynamics the expansion work is often called moving boundary work [1].

The objective is now to determine the moving boundary work of expanding universe assuming a quasiequilibrium expansion process. Consider that the system is experiencing a negative pressure $-\mathrm{P}$, and the pressure force acting on a differential area $d A$ is $-\mathrm{PdA}$ acts outward normal to the surface. Under the influence of the negative pressure the system will move outward a differential distance $d s$ and the system is undergoing a change in its volume equal to $d V=d A d s$, where $V$ is the total volume as shown in Figure 1. Noting that work is force times distance, then the boundary work done on the system is expressed by

$\delta W=-F d s=-\mathrm{P} d A d s=-\mathrm{P} d V$

Note that both $\delta$ and $d$ are used to indicate differential quantities, but $\delta$ is typically used for quantities that are path functions (such as work) and have inexact differentials, while $d$ is used for quantities that are point functions and have exact differential. Taking the integration of both sides of Equation (1) yields to $W=-\mathrm{P} V$

where $V$ is the net final total volume.

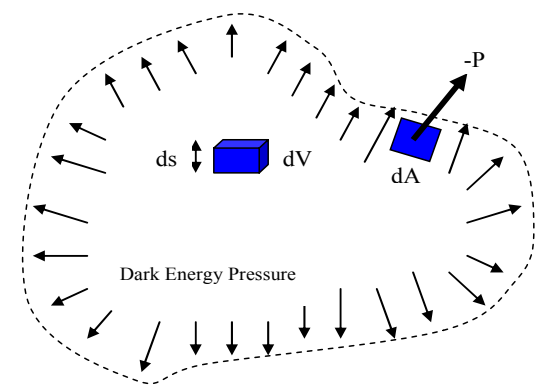

Figure 1: The Differential Surface of a Space-Time Universe System with Arbitrary Shape under the Influence of Expansion by the Negative Pressure of Dark Energy.

Defining the amount of energy exists per unit volume as the energy density $\rho$. Then, the amount of dark energy contained inside the universe system undergoing an increase in its volume is

$E=\rho V$

By the virtue of first law of thermodynamics of energy conservation and by comparing (2) with (3) yields

$\mathrm{P}=-\rho$

The physical interpretation of (4) is that the negative 
pressure that causing the universe to expand is equal to the negative of energy volumetric density. This force is behind the expansion of the universe.

Proof 2 (The Thermodynamics Equation of State of an Ideal Gas): This equation relates the pressure $P$, temperature $T$ and the volume $V$ of a substance behaves as an ideal gas [1], that is

$\mathrm{PV}=m R T=E$

Note that both sides of the equation has the units of energy (work done by pressure P). Assume now that dark energy behaves like an ideal gas with a negative pressure - $\mathrm{P}$ that causes the universe to expand with a total volume $V$, then by dividing both side of the equation of state (5) by $V$, it becomes

$\mathrm{P}=-\frac{m R T}{V}=-\frac{E}{V}=-\rho$

It is worth to mention that NASA's Cosmic Microwave Background Explorer (CMB) in 1992 estimated that the sky has a temperature close to $2.7251 \mathrm{Kelvin}$. Moreover, the Wilkinson Microwave Anisotropy Probe (WMAP) in 2003 has made a map of the temperature fluctuations of the CMB with more accuracy [10].

Proof 3 (The Cosmological Equation of State of a Perfect Fluid): The equation of state of a perfect fluid in cosmology is characterized by a dimensionless number $w$ [19], equal to the ratio of the pressure of the fluid to its energy density as follows

$\mathrm{P}=w \rho$

Recent observations of Lambda-Cold Dark Matter model (L-CDM) estimate the value of $w$ of a flat universe close to -1 , that is

$\mathrm{P}=-\rho$

Proof 4 (Invariant Variational Principle of Mechanics): The purpose of this proof is to interpret the nature of the negative pressure behind the expansion of the universe. Great advantage occurs sometimes through letting the time $t$ become one of the mechanical variables. Instead of considering the generalized position coordinates $\theta_{i}$ as a function of the time $t$, we consider the position coordinates $\theta_{i}$ and the time $t$ as mechanical variables, giving them as a function of some unspecified parameter $\zeta$. In relativistic mechanics this procedure is an absolute necessity since space and time are united into four-dimensional world of Einstein and Minkovski. Hence the Lagarngian configuration space has $N+1$ dimensions, we now add the time $t$ to the generalized coordinates $\theta_{i}$ as

$t=\theta_{N+1}$

Then, the corresponding phase space must comprise of $2 N+2$ corresponding to the $N+1$ pairs of canonical variables:

$$
\left(\begin{array}{l}
\theta_{1} \\
p_{1}
\end{array}\right),\left(\begin{array}{l}
\theta_{2} \\
p_{2}
\end{array}\right), \ldots,\left(\begin{array}{l}
\theta_{N} \\
p_{N}
\end{array}\right),\left(\begin{array}{l}
\theta_{N+1} \\
p_{N+1}
\end{array}\right)
$$

The system now has $N+1$ degrees of freedom (DOF). Denoting derivative with respect to $\varsigma$ by a prime "، ", the system action integral appears in the form

$$
W=\int_{\varsigma_{1}}^{\varsigma_{2}} L\left(\theta_{1}, \theta_{2}, \cdots, \theta_{N+1} ; \frac{\theta_{1}^{\prime}}{t^{\prime}}, \frac{\theta_{2}^{\prime}}{t^{\prime}}, \cdots, \frac{\theta_{N+1}^{\prime}}{t^{\prime}}\right) t^{\prime} d \varsigma
$$

For this purpose let the momentum associated with the time $t$ denoted as $p_{t} \equiv p_{N+1}$ be formed as

$$
\begin{aligned}
& p_{t}=\frac{\partial\left(L t^{\prime}\right)}{\partial t^{\prime}}=L-\left(\sum_{i=1}^{N} \frac{\partial L}{\partial \dot{\theta}_{i}} \frac{\theta_{i}^{\prime}}{t^{\prime 2}}\right) t^{\prime} \\
& p_{t}=L-\sum_{i=1}^{N} p_{i} \dot{\theta}_{i} \\
& p_{t}=-\left(\sum_{i=1}^{N} p_{i} \dot{\theta}_{i}-L\right)
\end{aligned}
$$

The last expression (14) is exactly the total energy of the system. This leads to the conclusion that the momentum associated with the time is the negative of the system total energy [26]. That is, every system is conserved when the time is considered a mechanical variable. In other words

$$
p_{t}=-E_{\text {TOTAL }}
$$

Noting that the momentum associated with the time as a mechanical variable has the units of work, considering that the system is expanding such that it has a total net volume $\Delta V$ and by dividing both side of (15) by $V$, one obtains

$\frac{p_{t}}{V}=-\frac{E_{\text {TOTAL }}}{V}$

Recalling that the work of the pressure done on the system as its volume changes is defined as $\mathrm{P}=W / V$, then (16) can be expressed as

$\mathrm{P}=-\rho$

The last equation is equivalent to Equation (4). The importance of Equation (17) is that it has the following physical interpretation: the momentum associated with time per unit volume (the pressure associated with time) is equal to the negative of energy density. In other words, it is the negative force behind the expansion of the universe.

Proof 5 (Euler-Lagrangian Equation): In addition to the previous analysis that has proved that the momentum associated with time is the dark energy, the objective now is to show that the flow of dark energy of the expanding universe is the momentum associated withe time. The Euler-Lagrangian equation takes the following form in case of a 4-D space-time world, which depends on the coordinates: $x=q_{1}, y=q_{2}, z=q_{3}, t=q_{4}$, 
$\operatorname{div} P_{\text {Total }}=\frac{\partial p_{1}}{\partial q_{1}}+\frac{\partial p_{2}}{\partial q_{2}}+\frac{\partial p_{3}}{\partial q_{3}}+\frac{\partial p_{4}}{\partial q_{4}}=0$

where $p_{i}$ is the momentum associated with coordinate

$q_{i}$. When dealing with a particle, only $t=q_{4}$ is

considered, and (18) is reduced to

$\frac{\partial p_{4}}{\partial q_{4}}=0$

Equation (19) implies that the time momentum is constant which by the virtue of (15) is interpreted as the system total energy, that is

$p_{4}=-E_{\text {Total }}=$ const.

Equation (19) suggests that $p_{4}$ is not a function of the time coordinate $t=q_{4}$.

Now by the virtue of Neother's Invariant Theorem and Divergence Transformation Theorem of Gauss [25-27], (19) can be interpreted as follows:

$\iint_{V} \int_{\operatorname{liv}} \operatorname{div} P d V=\iint_{S} P \cdot n d A$

where $P$ is the momentum vector of the 3-domensional space, $n$ is the normal vector to the surface $S$ and $d V$ is the segment of volume $V$. Now by considering that the momentum of time (as a mechanical variable) is constant and equals to the negative of the system total energy as in (20), then (21) can be modified as

$\iint_{S} P \cdot n d A+\frac{d}{d q_{4}} \iiint_{V} p_{4} d V=0$

or

$\iint_{S} P \cdot n d A=-\frac{d}{d q_{4}} \iiint_{V} p_{4} d V$

The physical interpretation of (23) is as follows: the flux of energy (work flow) flowing through the boundary surface of the expanding space-time universe is equal to the negative of the universe total energy (the momentum associated with time): $\mathrm{P}=-\rho$. In sense of the previous analysis an equivalent equation can be drawn from (18) as:

$$
\operatorname{div} P=-\frac{\partial p_{4}}{\partial q_{4}}
$$

Proof 6 (Einstein's Equation of General Relativity): The main goal of this proof is to show that Einstein's Cosmological constant Lambda is equivalent to the dark energy by applying Einstein's Equation of General Relativity. One of the candidates for dark energy is vacuum energy, or the Einstein cosmological constant, characterized by a pressure equal to the negative of the energy density [2][19]:

$R_{\mu v}-\frac{1}{2} R g_{\mu v}+\Lambda g_{\mu v}=8 \pi G T_{\mu v}$

where the left-hand side of Einstein's equation characterizes the geometry of the space-time and the right-hand side represents the energy sources, $g_{\mu \nu}$ is the space-time metric, $R_{\mu \nu}$ is the Recci tensor, $R$ is the space-time curvature scalar, $\Lambda$ is a free parameter called Einstein's cosmological constant (which equivalent to the dark energy), $G$ is the Newtonian Gravitational Universal Constant and $T_{\mu \nu}$ is the energy-momentum tensor . If the vacuum (space-time) is Lorentz-invariant, then the energy momentum will take the form

$T_{\mu v}^{v a c}=-\rho_{v a c} g_{\mu v}$

where $\rho_{\text {vac }}$ is the vacuum density (dark energy). Lorentzinvariant energy momentum is associated with an negative isotropic pressure such that

$\mathrm{P}_{\text {vac }}=-\rho_{\text {vac }}$

where $P_{v a c}$ is the vacuum pressure. It can be proved that Einstein's cosmological constant $\Lambda$ is proportional to dark energy so that

$\rho_{\text {vac }}=\frac{3 c^{2}}{8 \pi G} \Lambda$

The physical interpretation of (28) is that the dark energy density constant Lambda is equivalent to the cosmological constant Lambda by the constants $3 c^{2} / 8 \pi G$. Ongoing research by the author has proved that $\Lambda=0.52 \times 10^{-52} \mathrm{~m}^{-2}$ and $\rho_{\text {vac }}=1.28 \times 10^{-26} \mathrm{~kg} / \mathrm{m}^{3}$ and dark energy is a property of space-time itself.

Proof 7 (Einstein's Mass-Energy Equivalence Principle): Consider that the universe has a total energy $E_{\text {Total }}$ with an equivalent mass $M_{E q}$, then by the virtue of Einstein's Mass-Energy Equivalence Principle, the relation is given as

$E_{\text {Total }}=M_{E q} C^{2}$

where $C$ is the speed of light. By dividing the last equation by the universe volume, one gets

$\frac{E_{\text {Total }}}{V}=\frac{M_{E q}}{V} c^{2}$

or

$\rho=\rho_{E q, \text { mass }} c^{2}$

According to Equations (4), (6), (8), (23) and (27): $\mathrm{P}=-\rho$, then

$\mathrm{P}=-\rho_{E q, \text { mass }} c^{2}$

where $\rho_{E q \text {,mass }}$ is the equivalent dark energy mass density. Equation (31) shows that the pressure of the dark energy is equal to the negative of the equivalent dark energy mass density times the square of the light speed. 


\section{Can Dark Energy be Generated?}

Macro Scale: Astronomical observations have brought the evidence that dark energy is homogenous, isotropic and uniformly fills the space with a density approximated $10^{-26} \mathrm{~kg} / \mathrm{m}^{3}$. It is known that it does not interact with forces other than gravity. For example, for a space-time sphere with $1 \mathrm{~m}$ radius, the total amount of dark energy contained in it is $4.188 \times 10^{-26} \mathrm{~kg}$ which is equivalent to $3.7699 \times 10^{-9}$ Joules $(=23.562 \mathrm{GeV})$. One Joule of dark energy is equivalent to a space-time sphere of 642.5258 $\mathrm{m}$ radius. One kilo Joules of dark energy is equivalent to a space-time sphere of $6425.3 \mathrm{~m}$ radius. Meanwhile, One Mega Joules of dark energy is equivalent to a space-time sphere of $64252 \mathrm{~m}$ radius. Furthermore, One Gega Joules of dark energy is equivalent to a space-time sphere of $642.520 \mathrm{~km}$ radius. According to this analysis it seems that massive objects are needed to drag reasonable amounts of dark energy as galaxies, stars or planets as shown in Figure 2. Other factors should be tested to investigate their influence on dark energy such as the rotational speed of moving bodies and their temperatures. Objects with high rotational velocities will definitely increase their energies and as result more dark energy might be dragged. Massive objects with high surface temperatures might as well attract more dark energies.
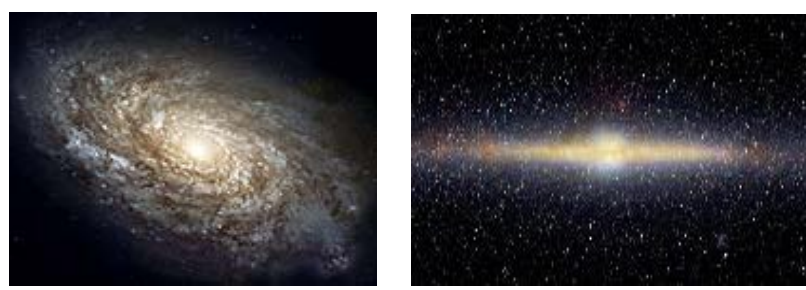

Figure 2: Spiral Galaxy and the Milky Way Galaxy (NASA's Hubble Telescope)

Micro-Nano Scale: Previous analysis is based on the macro scale level. To test now the existence of dark energy at the micro-nano level, the following experiment is proposed. This system is composed of a closed cubic box of edge length equal to $1 \mathrm{~m}$ and a micro pistoncylinder is then to be placed inside that box. Such a micro piston-cylinder might be fabricated using Micro-ElectroMechanical Systems technology (MEMS). The total amount of dark energy contained inside the cubic box is in the range of $10^{-26} \mathrm{~kg}$ which is equivalent to $0.90 \times 10^{-9}$ Joules (or $5.625 \mathrm{GeV}$ ). If the volume of the micro pistoncylinder is chosen such that it has a $1 \mathrm{~cm}^{3}$, it contains an amount of energy equivalent $10^{-32} \mathrm{~kg}$ or $9 \times 10^{-16}$ Joules or $5625 \mathrm{eV}$. Now if the micro piston-cylinder is placed inside the cubic box, then the piston will move to the left as shown in Figure 3. That is because the dark energy inside the cubic box is much higher that that inside the micro piston-cylinder. Such a displacement is proportional to the dark energy density. The work of the dark energy is $\delta W=-F d s=-\mathrm{PAds}$ as in (1). According to the principle of conservation of energy, this work should be equal to the kinetic energy of the piston $K E=0.5 m_{p}(d s)^{2}$. Hence, $-\mathrm{PAds}=0.5 m_{p}(d s)^{2}$, then, $d s=-2 \mathrm{PA} / m_{p}$, where $m_{p}$ is the piston mass, if the piston area is chosen as $A=10^{-4} \mathrm{~m}^{2}$, its mass is $10^{-3} \mathrm{~kg}$, and estimating the dark energy pressure as $0.90 \times 10^{-9} \mathrm{~J} / \mathrm{m}^{3}$ $(5.625 \mathrm{GeV})$, then the piston will move 0.18 nanometer. By this, it is possible that dark energy can be utilized to operate mico or nano systems. Such systems can have several applications in space, biomedical or electronics applications.

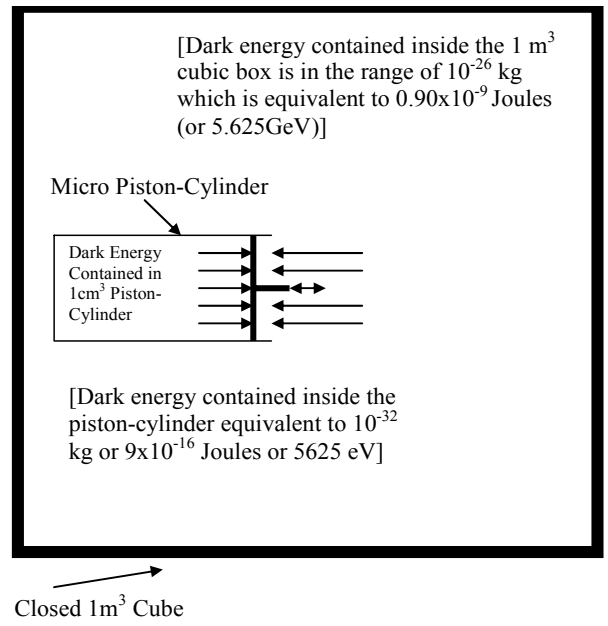

Figure 3: Micro Piston-Cylinder System to Test the Pressure Work of Dark Energy: [The piston will move by 0.18 nano meter by effect of the negative force of dark energy].

\section{Simulation Results}

The purpose of simulation is to show the dark energy associated with a mechanical system by considering time as a mechanical variable. The dark energy is equal to the momentum associated with time. Such energy (negative pressure) drifts the system a way. The a system is composed of 6-DOF micro space robot arm and mounted on a micro base satellite and used to demonstrate the analytical results. The mass of the base servicing satellite is chosen as $10 \mathrm{~g}$, the masses of the 6-robot arm as [ $\left[\begin{array}{lll}1 & 1 & 1\end{array}\right.$ $\left.\begin{array}{lll}1 & 1 & 1\end{array}\right]$ g. All initial conditions are assumed to be zero. The desired values for the robot angular position are chosen as $q_{\text {des }}=[0.30 .20 .10 .60 .50 .4$ ]'. A PD controller is used to control the micro space-satellite robot with 0.01 value selected for both the proportional and derivative parameters.

The simulation in Fig. 4 shows the momentum associated with the time by considering it as a mechanical variable (dark energy), meanwhile, Fig. 5 shows a comparison between the classical momentum and the drift in momentum by considering time as a mechanical variable. This comparison indicates that there is indeed a drift (expansion) in the momentum generated by time as a mechanical variable and this drift is equal to the negative of the system total energy (dark energy). The drift in Figure 5 at the selected points for example is equal to $5.2600 \times 10^{-5}$ Joules and this value is relatively significant at the micro scale. 


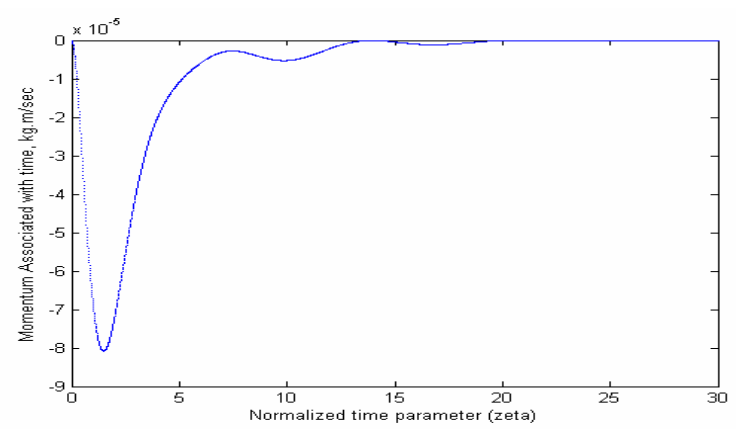

Figure 4: Dark Energy Associated with Time as a Mechanical Variable of a 6 DOF Micro Space Robot $\left(10^{-5} \mathrm{~J}\right)$

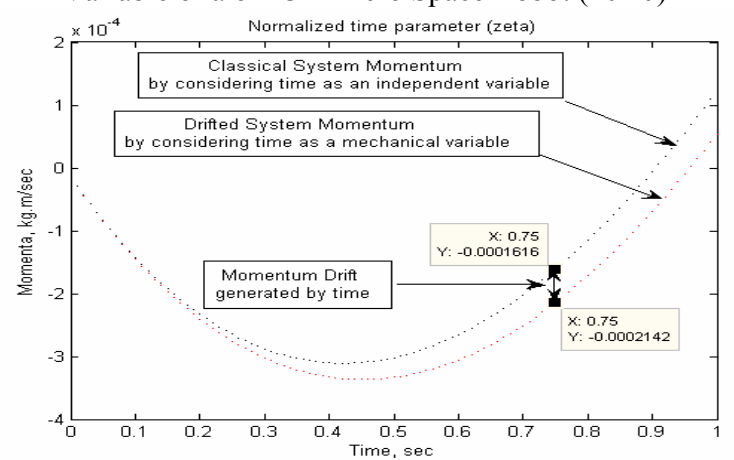

Figure 5: Drifted Momentum (Dark Energy Effect) by Considering Time as a Mechanical Variable of a 6 DOF Micro Space Robot $\left(10^{-4} \mathrm{~J}\right)$

\section{Conclusions}

A foundation of the fourth law of thermodynamics and dark energy is proposed. Such a law presents an explanation of the expansion of the universe by the negative pressure generated by the momentum associated with time as a mechanical variable. Seven independent proofs are presented. Generation of dark energy by massive objects or by micro-nano systems is discussed and finally simulation results re demonstrated.

\section{Acknowledgment}

The author would like to acknowledge his Excellency Prof. Dr. Labib Khadra, the President of the German Jordanian University for supporting the research and providing a great atmosphere at the university.

\section{References}

[1] Y. A. Cengel, M. A. Boles, Thermodynamics: an Engineering Approach, Fifth Edition, McGraw Hill, 2006.

[2] A. Einstein, "The Foundation of the General Theory of Relativity," pp., 146-200, [The collected papers of Einstein, English Translation Edited by A.J. Kox, M.J. Klien, and R. Schulmann, Volume 6, Princeton University Press, 1997], The Berlin Years Writings, 1914-1917.

[3] R. A. Knop, G. Aldering, R. Amanullah, et al., "New Constraints on $\Omega_{M}, \Omega_{\Lambda}$, and $\Omega_{\Lambda}$ from an Independent Set of Eleven High-Redshift Supernovae Observed with HST1," The Astrophysical Journal, Sep 12, 2003.

[4] S. Permutter et al., "Measurements of Omega and Lambda from 42 High Redshift Supernovae," Astrophysical Journal, pp. 565-86, 1999.
[5] A. G. Riess et al., "Observational Evidence from Supernovae for an Accelerating Universe and Cosmological Constant," Astronomical Journal, pp. 1009$1038,1998$.

[6] R. Maartens and E. Majerotto, "Observational Constraints on Self-Accelerating Cosmology," Journal of Astrophysics, June 14, 2006.

[7] S. Perlmutter, M.S. Turner and M. White, "Constraining Dark Energy with SNe Ia and Large-Scale Structure," Journal of Astrophysics, Jan 15, 1999.

[8] W. L. Freedman et al., "Final Results from the Hubble Space Telescope Key Project to Measure the Hubble Constant," Journal of Astrophysics, Dec 18, 2000.

[9] J. L. Tonry et al., "Cosmological Results from High-z Supernovae," Journal of Astrophysics, May 1, 2003.

[10] D. N. Spergel et al., "Willkinson Microwave Anisotropy Probe (WMAP) three years results: implications for cosmology," NASA publications, March 2006.

[11] M. S. Turner, "Dark Matter and Dark Energy in the Universe," The Third Astro. Symposium: The Galactic Halo ASP Conference Series, Vol. 666, 1999.

[12] D. Huterer and M. S. Turner, "Prospects for probing the dark energy via supernova distance measurements," Physical Review D, Vol. 60, 1999.

[13] S. M. Carroll, Mark Hoffman, Mark Trodden, "Can the dark energy equation-of-state parameter $\mathrm{w}$ be less than 1?," Physical Review D 68, 2003.

[14] D. Huterer and Michael S. Turner, "Probing dark energy: Methods and strategies," Physical Review D, Vol. 64, 2001.

[15] P. J. E. Peebles and Bharat Ratra, "The cosmological constant and dark energy," Reviews of Modern Physics, Vol. 75, April, 2003.

[16] E. J. Copeland, M. Sami, and S. Tsujikawa, "Dynamics of Dark Energy," International Journal of Modern Physics, 16 June, 2006.

[17] G. E. Volovik, "Vcauum Energy: Myths and Reality," International Journal of Modern Physics A, 16 June, 2006.

[18] S. M. Carroll, I. Sawicki, A. Silvestri, and M. Trodden, "Modified-Source Gravity and Cosmological Structure Formation," Journal of Astrophysics, July 19, 2006.

[19] S. M. Carroll, "Why is the Universe Accelerating?," Journal of Astrophysics, Nov. 18, 2003.

[20] S. M. Carroll, "The Cosmological Constant" Living Reviews of Relativity, 2001, retrieved on 2006.

[21] G. Dvali and M. Turner, "Dark Energy as a Modifications of the Friedmann Equation," Journal of Astrophysics, Jan. $25,2003$.

[22] M. Shibli, "Physical Insight of Universe Dark Energy: the Space Mission," 1st IEEE International Symposium on Systems and Control in Aerospace and Astronautics (ISSCAA 2006), Co-sponsored by AIAA, Harbin, China, Jan. 19-21, 2006.

[23] M. Shibli, "Canonical Modeling Approach of a Micro/Nano Free-Flying Space Robot: A Proposal towards Detecting the Nature of Space-Time," 1st IEEE ISSCAA, Co-sponsored by AIAA, Harbin, China, Jan. 19-21, 2006.

[24] M. Shibli, "Physical Insight of Space-Time and Modeling of Space-Time Dipoles, Gravity Waves and Gravitons: A Micro Space Antenna to Detect the Nature of Gravity Waves"," 1st IEEE International Conference on Advances in Space Technologies (ICAST2006), Pakistan, Sep $2^{\text {nd }}-4^{\text {th }}$, 20062006.

[25] H. Goldstien, Classical Mechanics, $2^{\text {nd }}$ ed., AddisonWesley, 1980.

[26] C. Lanczos , The Variational Principle of Mechanics, University of Toronto Press, Toronto, 1966.

[27] E. Noether, "Inavariante Variationsprobeleme," Goett. Nochr. , pp. 235-257, 1918. 